\title{
Profiles of patients' self-reported health after acute stroke
}

\author{
D. Leander Rimmele ${ }^{1 *}$ (D) Theresa Schrage ${ }^{2}$, Lisa Lebherz ${ }^{2}$, Levente Kriston ${ }^{2}$, Christian Gerloff ${ }^{1}$, Martin Härter ${ }^{2}$ and \\ Götz Thomalla'
}

\begin{abstract}
Background: We aimed to identify groups of patients with similar health status after stroke, assessed by patient reported outcome measures (PROMs), to improve initial risk stratification.

Methods: In a prospective study, inpatients were recruited during acute stroke treatment. Demographics, history, and cardio-vascular risk factors were assessed at baseline. Self-reported functional status, physical and mental health as well as anxiety and depressive symptoms were assessed 3 and 12 months after stroke and used to identify latent classes. The association of patient characteristics with latent class membership was investigated with multinomial logistic regression.

Results: Of the 650 patients included with a mean age of 75 years and $48 \%$ female, $70 \%$ had ischemic, $6 \%$ hemorrhagic strokes, and 24\% transient ischemic attacks. Median NIHSS on admission was 2 (IQR:0,5). Values of PROMs remained comparable at 3 and 12 months. A three-class model was developed, differentiating between patients with mildly (75\%), moderately (17\%), and severely (8\%) impaired self-reported health status. Adjusted for univariately significant baseline characteristics, initial NIHSS distinguished mild- from moderate-, and moderatefrom severe-class-membership $(p<0.001)$. Length of inpatient stay $(p<0.001 ; \mathrm{OR}=1.1)$, diabetes $(p=0.021 ; \mathrm{OR}=$ 1.91 ), and atrial fibrillation ( $p=0.004 ; \mathrm{OR}=2.20$ ) predicted allocation to the moderately vs. mildly affected class.
\end{abstract}

Conclusions: Grading stroke patients by a standard set of PROMs up to 1 year after stroke allows to distinguish the diverse impact of baseline characteristics on differently affected groups. In addition to initial stroke severity, longer inpatient stay, presence of diabetes and atrial fibrillation correlate with greater impairment of self-reported health in the less affected groups.

Trial registration: http://www.ClinicalTrials.gov; Unique identifier: NCT03795948.

Keywords: Stroke, Patient-reported outcome measures, Value-based health care, ICHOM, PROMIS

\section{Introduction}

Prevention and improved treatment of acute stroke has led to a decline in stroke incidence and stroke-related mortality over the past decades. The global burden of stroke, however, remains high due to the prevalence of its chronic stage [14]. Disability caused by stroke may affect a broad range of functions from motor to

\footnotetext{
* Correspondence: d.rimmele@uke.de

${ }^{1}$ Department of Neurology, University Medical Center Hamburg-Eppendorf, Martinistr. 52, 20246 Hamburg, Germany

Full list of author information is available at the end of the article
}

neuropsychological deficits [7]. With the aim of prevention and intervention, different questionnaires have been developed to characterize and predict the impact of stroke on functional status and quality of life. To this end, a systematic assessment of patient reported outcomes measures (PROMs) has been shown to deliver information beyond routine examinations by physicians and to identify disabling deficits in every-day life after stroke $[17,18]$. A possible strategy towards understanding the development of different outcomes and towards risk stratification is classifying patients by the results of

(c) The Author(s). 2021 Open Access This article is licensed under a Creative Commons Attribution 4.0 International License, which permits use, sharing, adaptation, distribution and reproduction in any medium or format, as long as you give appropriate credit to the original author(s) and the source, provide a link to the Creative Commons licence, and indicate if changes were made. The images or other third party material in this article are included in the article's Creative Commons licence, unless indicated otherwise in a credit line to the material. If material is not included in the article's Creative Commons licence and your intended use is not permitted by statutory regulation or exceeds the permitted use, you will need to obtain permission directly from the copyright holder. To view a copy of this licence, visit http://creativecommons.org/licenses/by/4.0/. 
their outcomes and analyzing class characteristics, as shown for diabetes [24] and heart transplantation [20]. In mild to moderately affected stroke patients from an outpatient setting, a retrospective analysis showed promising results building distinct profiles or classes using the Patient-reported Outcomes Measurement Information System (PROMIS) [16]. The International Consortium for Health Outcome Measurement Standard Set for Stroke (ICHOM-SSS) has been developed to standardize patient reported outcomes after stroke [30]. We have recently assessed its use in clinical routine at our hospital [27] and amended the Patient-Health-Questionaire-4 (PHQ-4) to address reported depressive symptoms and anxiety after stroke and transient ischemic attacks (TIA). Considering evidence of comparable risk profiles [31] and similar self-reported outcomes in patients with acute stroke and TIA, we decided to assess both, in order to adequately represent the heterogenous stroke unit clientele $[4,16]$. We aimed to grade this unselected clientele of patients recruited in a prospective observational study on stroke unit admission and identify latent classes according to health status assessed by PROMS 3 and 12 months after the event.

\section{Methods}

\section{Study design}

The study was designed as a prospective observational analysis for an exploratory longitudinal evaluation of patients with the ICHOM-SSS. Patients were recruited over a 15 month period during initial treatment on the stroke unit of the University Medical Center HamburgEppendorf after diagnosis of acute ischemic stroke (AIS), transient ischemic attack (TIA), intracerebral hemorrhage $(\mathrm{ICH})$, or retinal infarction. Patient-reported outcomes were assessed at follow-up 3 and 12 months after admission. Informed consent was obtained by patients or authorized guardians. The ethics committee of the Hamburg chamber of physicians approved the study protocol (PV54565). The study was registered at ClinicalTrials.gov, NCT03795948, at January 82,019 retrospectively.

\section{Data acquisition}

Data were collected according the ICHOM guidelines and the study protocol $[11,28]$. Diagnosis, patient history, demographics, cardio-vascular risk factors, and therapy were collected from interviews and electronic records prior to discharge. The National Institutes of Health Stroke Scale (NIHSS) was examined at admission. Cardiovascular risk factors were defined by presence of relevant diagnoses in medical records or determined by interview. Follow-up assessments of patient-reported outcomes were done at 3 and 12 months after admission by pen-and-paper questionnaire and telephone. They included questions concerning living situation, living location, and recurrent stroke events. Details of assessment have been described previously [27].

\section{Outcomes}

The ICHOM-SSS is based on the PROMIS 10-Question Short Form (PROMIS-10), which contains four items each for mental and physical health used to calculate Tscores. Lower values indicate a poorer outcome. The reference mean is 50 with a standard deviation (SD) of 10 measured in the general population [5].

Functional status post stroke was assessed by one question each for dependence on the help of others for walking (yes, partly, no), dressing (yes, no), and toileting (yes, no), with 0 representing fully independent (high functional status) and 4 fully dependent (low functional status) [11].

We administered the Patient Health Questionnaire-4 (PHQ-4) in addition to the ICHOM-SSS to address symptoms of anxiety and depression. It consists of two items each for potential presence of anxiety and depressive symptoms. Scales for each reach from one to six with values of three and above indicating anxiety or depressive symptoms. Reference mean values of a general population for anxiety are $0.82(\mathrm{CI}: 0.79 ; 0.85)$ and for depressive symptoms 0.94 (CI:0.90;0.97) [21].

\section{Statistical analysis}

All patients with the diagnosis of AIS, TIA, or ICH and at least one valid measurement of PROMIS-10, functional status, or PHQ-4 at 3 or 12 months were included in the analysis. We excluded patients with retinal ischemia from analysis due to different clinical manifestation.

Descriptive and multinomial regression analyses were carried out using IBM SPSS Statistics, Version 25.0 (Armonk, NY: IBM Corp), and JASP Version 0.14. The latent profile analysis was performed in Mplus version 7.2 (Muthén \& Muthén, Los Angeles, CA).

\section{Latent profile analysis}

We performed a latent profile analysis (LPA) based on patient-reported health data to identify groups of similar outcome [23]. Patient-reported assessments of physical and mental health, functional status, depressive symptoms, and anxiety 3 and 12 months after acute stroke treatment were used to characterize the groups respectively classes. Class membership was defined by a series of models varying across three dimensions. First, withinclass correlations between the analyzed variables were modeled in three different ways: all correlations restricted to be zero, non-zero correlations only between the same variables measured at different timepoints, and non-zero correlations allowed between any of the variables. Second, within-class variance of the same variables measured at different timepoints were either restricted 
to be equal or allowed to be different. Third, the number of classes were varied from one to six. This results in a total of $3 \times 2 \times 6=36$ estimated models. We use a robust maximum likelihood estimator with 20,000 starting values for fitting the models. Model selection criteria and fit indices are reported in the supplementary.

\section{Multinominal regression}

After determining the most likely class membership for each patient, we investigated the association of demographic, clinical, and functional parameters with class membership using multinomial logistic regression. The class of mild impairment was opposed to the one of moderate and the class of moderate impairment to severely impaired separately. Binary univariate regression was performed in advance, and parameters associated with class membership with $p<0.05$ were included in multivariable multinominal logistic regression models. The NIHSS was used in categories of no (0), mild (1-4), moderate (5-15), moderate to severe (16-21) and severe (22-42) symptoms.

\section{Results}

\section{Patient characteristics and self-reported outcomes}

We included 650 patients in the analysis. $70 \%$ of them were diagnosed having acute ischemic stroke, $6 \%$ intracranial hemorrhage, and $24 \%$ transient ischemic attack. Mean age was 75 years, $48 \%$ were female, and the median NIHSS assessed on admission was 2.0 (IQR:0,5). $67 \%$ of patients lived without need of external support, and 59\% lived with a partner. Categorized by the NIHSS, 29.5\% had no neurological deficit (NIHSS: 0), 41.8\% mild deficits (NIHSS: 1-4), 23.7\% moderate deficits (NIHSS: 5-15), 3.4\% moderate to severe deficits (NIHSS: 16-20), and $1.5 \%$ severe deficits (NIHSS: 21-42). Concerning risk factors, $62 \%$ had hypertension, $19 \%$ atrial fibrillation, and $15 \%$ diabetes.

Their mean reported physical health after 3 and 12 months of follow up measured by PROMIS-10 was 44.7 $(\mathrm{SD}=10.00, n=555)$ and $44.9 \quad(\mathrm{SD}=10.19, n=516)$. Mean mental health scores were $41.0(\mathrm{SD}=9.73, n=$ $562)$ and $41.6(\mathrm{SD}=10.05, n=522)$, respectively. Values of physical and mental health were reduced compared to their reference value of $50(\mathrm{SD}=10)$ from healthy subjects, with lower values representing increased impairment. Scores of the PHQ-4 after 3 months indicated anxiety in $16.3 \%(n=90 / 552$; mean $=1.35 \pm 1.42)$ and depressive symptoms in $17.4 \% \quad(n=96 / 553$; mean $=$ $1.46 \pm 1.45)$ of the patients. After 12 months $16.3 \%(n=$ $82 / 502$; mean $=1.33 \pm 1.50)$ scored indicative for anxiety and $19.1 \%(n=96 / 503$; mean $=1.50 \pm 1.53)$ for depressive symptoms. Functional impairment post-stroke determined by the ICHOM-SSS had a mean value of 0.45
( $\mathrm{SD}=1.00, n=568)$ after three, and of $0.47(\mathrm{SD}=0.98$, $n=529)$ after 12 months.

\section{Latent classes}

According to the information criteria, models with freely estimated within-class correlations between variables and freely estimated within-class variances showed the best fit to the data (Supplemental Table I). Among them, only models with one to four classes had at least 30 participants in the smallest class. Of these four models, information criteria supported the four-class model, while the likelihood ratio tests supported the two-class solution. Therefore, we investigated the models with two, three, and four classes further in the class evolution tree analysis, which suggested a successive differentiation according to the severity of impairment as assessed by patient-reported outcome measures (Fig. 1). In summary, the latent profile analysis suggested a roughly unidimensional classification scheme with the severity of impairment of one's health being largely independent of the specific health domains and allowing for a differentiation between two, three, or four grades of impairment.

In the two-class model, $576(88.6 \%)$ patients showed milder and $74(11.4 \%)$ more severe impairment. In the three-class model, 487 (74.9\%) patients reported of mild, $112(17.2 \%)$ of moderate, and 51 (7.8\%) of severe deficits (Fig. 2). Division in four classes led to 490 (75.4\%) patients, who reported of mildly impaired, 86 (13.2\%) of moderately, $42(6.5 \%)$ of moderately to severely, and 32 (4.9\%) of severely impaired health. For further investigation, we focused on the three-class model, because it offers a sufficiently differentiated but still practical classification with divisible severity and class size. Patients' characteristics in the three-class model are displayed in Table 1.

\section{Characteristics associated with class membership}

Assessed in univariate comparison of one class with one other (Supplemental Table II), the average age continuously increased from 71 years in the mildly affected class one to 75 years in the moderately affected class two ( $p=$ 0.005 ; OR $=1.03$ ), and from class two further to 79 years in the severely affected class three $(p=0.046$; $\mathrm{OR}=1.03)$. The severity of initial neurological deficits assessed by the NIHSS paralleled worse PROs with a median of 4 (IQR:2,9) in the class of moderately affected PROs compared to a median of 1 (IQR:0,3.5) in the mildly affected class $(p<0.001 ; \mathrm{OR}=2.30)$, and a median of 14 (IQR: $6,16)$ in the most severely opposed to 4 (IQR:2,9) in the moderately affected class $(p<0.001 ; \mathrm{OR}=3.19)$. Length of initial inpatient stay showed an increase from mildly to the moderately affected class $(p<0.001 ; \mathrm{OR}=1.10)$. Stroke subtype differed between these classes as well $(p<0.001)$, as did cardiovascular risk factors: In the class 


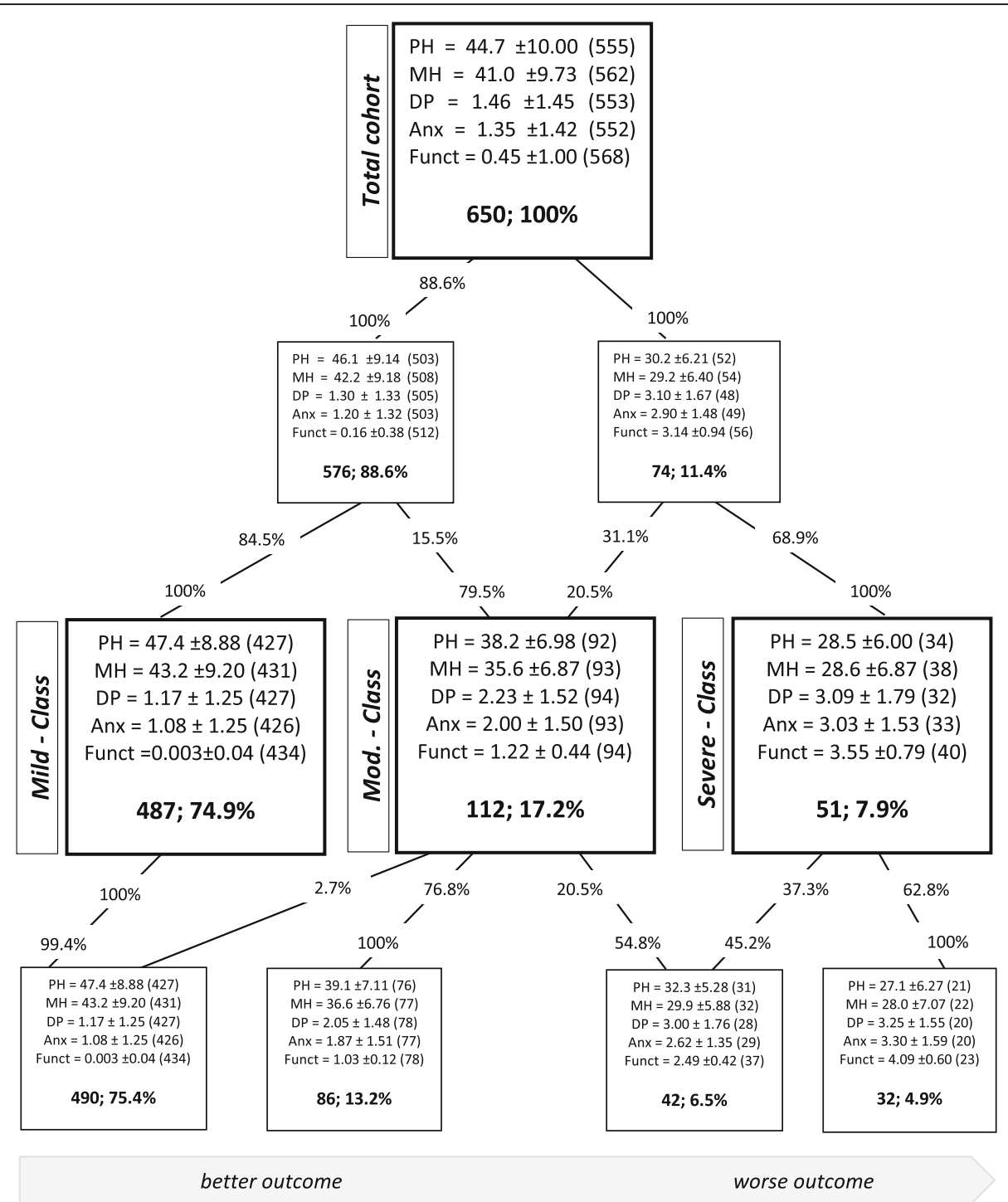

Fig. 1 Class Evolution Tree of the four empirically most strongly supported models from the latent profile analysis. The bold numbers indicate absolute and relative frequency. The depicted variables are patient-reported outcomes. Abbreviations: $\mathrm{PH}=$ physical health; $\mathrm{MH}=$ mental health; Dep $=$ depressive symptoms; $A n x=$ anxiety; Funct $=$ functional status; mod. $=$ moderate

of mild impairment, $13 \%$ had diabetes compared to $24 \%$ in the class of moderate impairment $(p=0.002 ; \mathrm{OR}=$ $2.19)$, the rate of atrial fibrillation was $15 \%$ compared to $32 \%(p<0.001$; OR $=2.70)$, and $7 \%$ had prior MI compared to $13 \%(p=0.037 ; \mathrm{OR}=2.03)$. The class of moderate and severe impairment differed in the percentage of patients living alone $(p=0.010 ; \mathrm{OR}=2.53)$, a higher rate of endovascular thrombectomy $(p<0.001 ; \mathrm{OR}=1.85)$ and women being more frequent in the severely than the moderately affected class $(p=0.041 ; \mathrm{OR}=2.04)$.

The multivariate model of 595 patients belonging to the class of mild or moderate impairment in PROs showed that initial severity of neurological deficits $(p<0.001 ; \mathrm{OR}=1.74)$, length of initial inpatient stay $(p<0.001 ; \quad$ OR $=1.08)$, atrial fibrillation $(p=0.004$;
$\mathrm{OR}=2.20)$, and diabetes $(p=0.021 ; \mathrm{OR}=1.91)$ predicted membership to the moderately affected class (Table 2).

Comparing membership of the class of moderate with the class of severe impairment in a model of 158 patients, only initial severity of neurological symptoms $(p<0.001 ; \mathrm{OR}=3.51)$ was significantly associated with membership to class three (Table 3 ).

\section{Discussion}

In our study of consecutive stroke patients treated in clinical practice, grading of patients by classes of PROMs up to 12 months after stroke identified different profiles of patients' demographic and clinical characteristics. Class membership was associated with stroke severity, 


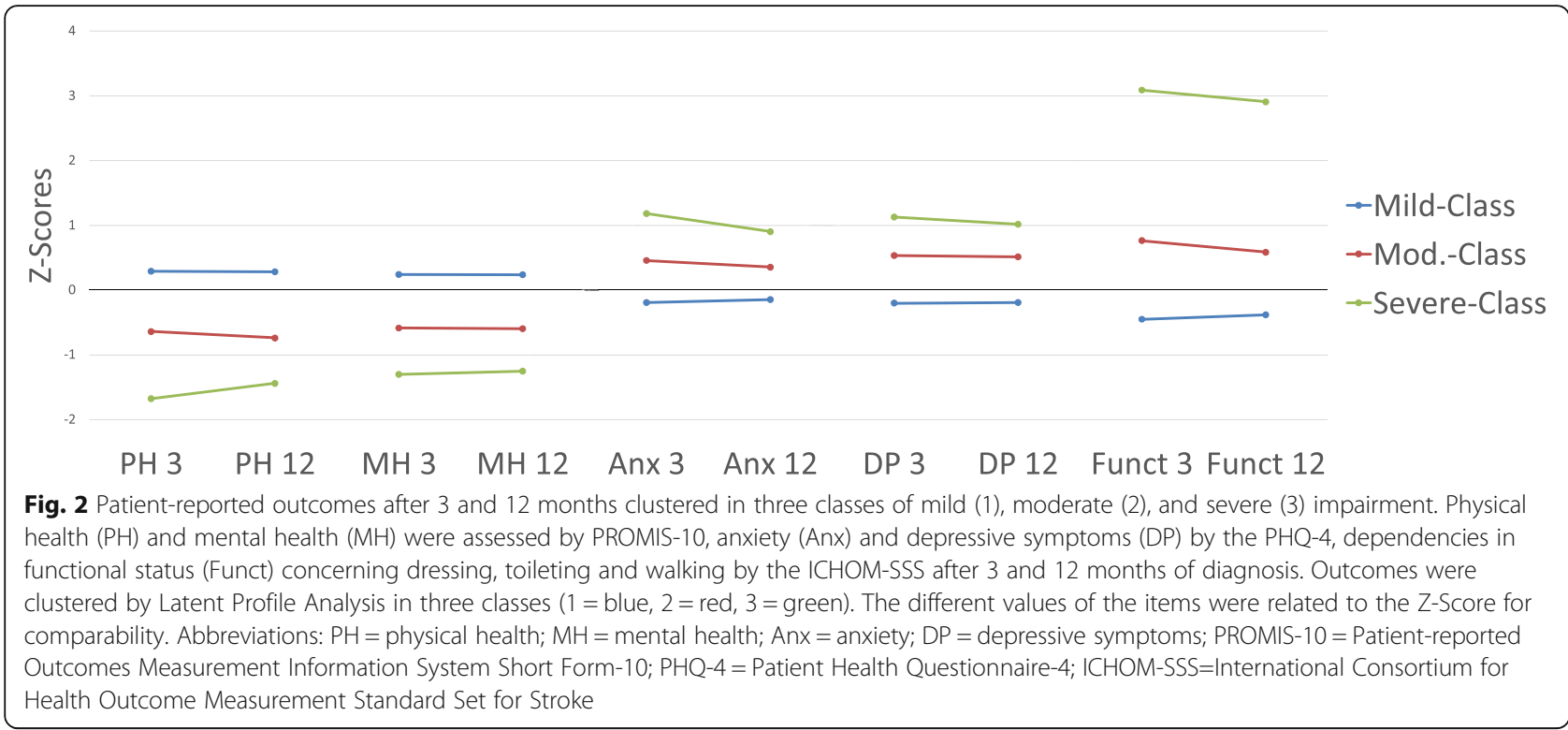

stroke subtype, pre-stroke living situation, and cardiovascular comorbidities to a different degree. In a threeclass-model, the severity of initial neurological deficits was the only predictor of class membership across all three classes from patients with only minor impairment to those with severely impaired health status. In addition, history of diabetes or atrial fibrillation, and length of in-hospital stay predicted assignment to the class of moderately impaired health status instead of the class of mild impairment.

The cohort studied here consists of unselected patients from a university stroke center admitted as inpatients and followed prospectively after acute treatment. With a mean of 75 years, high frequency of cardiovascular risk factors, and the observed distribution of stroke subtypes, our cohort is representative for the general stroke population $[10,26]$. In this sense, our study adds new data to class analysis of stroke outcome based on PROMs, as the only published comparable was a retrospective analysis comprised of younger patients with overall less severe impairment [15]. We used the ICHOM-SSS with its standardized selection of PROMs to assess its value in routine care and enable comparability. The parameters recorded with the ICHOM-SSS and PHQ-4 provided sufficient information to calculate class models with a plausible distribution and reflection of patient characteristics as compared to the previous class analysis after stroke [15]. The frequency of patients with scores indicative for anxiety and depressive symptoms matches previous analyses as well [4]. In our study, absolute values of PROMs and class differences remained rather stable between 3 and 12 months after stroke. This observation stands in contrast to previously reported relevant improvements of externally rated outcomes using the modified Ranking Scale or Barthel index in the same time-frame and might indicate the different scope of PROMs as compared to assessments by health professionals $[9,17]$.

The differences of each patient class to one another in severity of neurological deficits in our models are similar to the ones described in the retrospective analysis of an outpatient setting, and the association of worse values of PROMs after stroke with greater initial neurological deficits has been described before [1,33]. Beyond initial stroke severity, history of diabetes and atrial fibrillation predicted assignment to the moderately affected instead of the mildly affected class. This indicates that treatable cardiovascular comorbidities are especially relevant for self-reported outcomes of patients with mild to moderate initial neurological deficits. Negative self-affirmation in controlling their comorbidity following the stroke event might in the mild to moderately affected groups not be outweighed by severe neurological deficits and the reason for the influence of comorbidity on PROMs $[2,13]$. These findings might thus point to new treatment targets after stroke as psychotherapeutic support in handling of comorbidities. In support of this judgement, the Northern-Manhattan-Study found that diabetes predicts decline in self-assessed Barthel index [6].

Length of initial inpatient stay independently predicted membership of the moderately affected class opposed to the mildly. This is in line with data showing that patients with moderate neurological deficits benefit most from early supportive discharge $[3,19]$. The clearer characterization of the moderate outcome class may help in identifying patients that could benefit from early 
Table 1 Distribution of patients' characteristics in the three-class model

\begin{tabular}{|c|c|c|c|}
\hline Variable & $\begin{array}{l}\text { Mild-Class } \\
N=487\end{array}$ & $\begin{array}{l}\text { Moderate-Class } \\
N=112\end{array}$ & $\begin{array}{l}\text { Severe-Class } \\
N=51\end{array}$ \\
\hline Age, mean (SD) & $70.84(12.76)$ & $74.62(12.02)$ & $78.61(10.48)$ \\
\hline \multicolumn{4}{|l|}{ Gender } \\
\hline Female & $224(46.0 \%)$ & $53(47.3 \%)$ & $33(64.7 \%)$ \\
\hline Male & $263(54.0 \%)$ & $59(52.7 \%)$ & $18(35.3 \%)$ \\
\hline Living situation: alone & $174(35.7 \%)$ & $38(33.9 \%)$ & $26(56.5 \%)$ \\
\hline \multicolumn{4}{|l|}{ Living location (at 90d) } \\
\hline At home without external support & 381 (88.2\%) & $54(57.4 \%)$ & $3(7.7 \%)$ \\
\hline \multicolumn{4}{|l|}{ Stroke type } \\
\hline Acute ischemic stroke & $323(66.3 \%)$ & $91(81.3 \%)$ & $41(80.4 \%)$ \\
\hline Intracranial hemorrhage & $22(4.5 \%)$ & $9(8.0 \%)$ & $9(17.6 \%)$ \\
\hline Transient ischemic stroke & $142(29.2 \%)$ & $12(10.7 \%)$ & $1(2.0 \%)$ \\
\hline \multicolumn{4}{|l|}{ Lesion area } \\
\hline Hemisphere left/right (\%) & $222 / 163$ & $52 / 42$ & $23 / 23$ \\
\hline Brainstem & $89(18.3 \%)$ & $16(14.3 \%)$ & $3(5.9 \%)$ \\
\hline Both hemispheres or brainstem+ & $12(2.5 \%)$ & $2(1.9 \%)$ & $2(3.9 \%)$ \\
\hline MRI at initial inpatient stay & $188(38.6 \%)$ & $43(38.4 \%)$ & $12(23.5 \%)$ \\
\hline Stroke severity [NIHSS] median (IQR) & $1(0 ; 3.5)$ & $4(2 ; 9)$ & $14(6 ; 16)$ \\
\hline No stroke symptoms [0] & $182(37.4 \%)$ & $10(8.9 \%)$ & $0(0.0 \%)$ \\
\hline Mild stroke symptoms [1-4] & $212(43.5 \%)$ & $49(43.8 \%)$ & $11(21.6 \%)$ \\
\hline Mod. stroke symptoms [5-15] & $81(16.6 \%)$ & $48(42.9 \%)$ & $25(49.0 \%)$ \\
\hline Mod. to sev. Stroke symptoms [16-21] & $8(1.6 \%)$ & $4(3.6 \%)$ & $10(19.6 \%)$ \\
\hline Severe stroke symptoms [21-42] & $4(.8 \%)$ & $1(.9 \%)$ & $5(9.8 \%)$ \\
\hline \multicolumn{4}{|l|}{ Stroke treatment } \\
\hline Thrombolysis & $88(18.1 \%)$ & $25(22.3 \%)$ & $13(25.5 \%)$ \\
\hline Endovascular thrombectomy & $49(14.9 \%)$ & $16(17.4 \%)$ & $19(46.3 \%)$ \\
\hline \multicolumn{4}{|l|}{ Cardio-vascular risk factors } \\
\hline Prior ischemic stroke & $81(16.6 \%)$ & $22(19.8 \%)$ & $10(20.0 \%)$ \\
\hline Prior transient ischemic stroke & $18(3.7 \%)$ & $6(5.4 \%)$ & $0(0.0 \%)$ \\
\hline Prior myocardial infarction & $32(6.6 \%)$ & $14(12.5 \%)$ & $1(2.0 \%)$ \\
\hline Coronary artery disease & $57(11.8 \%)$ & $7(6.3 \%)$ & $6(12.0 \%)$ \\
\hline Atrial fibrillation & $71(14.6 \%)$ & $35(31.5 \%)$ & $17(34.0 \%)$ \\
\hline Diabetes mellitus & $62(12.8 \%)$ & $27(24.3 \%)$ & $11(22.0 \%)$ \\
\hline Hypertension & $290(59.9 \%)$ & $75(67.6 \%)$ & $37(74.0 \%)$ \\
\hline Hyperlipidemia & $67(14.2 \%)$ & $14(12.8 \%)$ & $3(6.3 \%)$ \\
\hline Smoking & $100(21.8 \%)$ & $18(18.6 \%)$ & $4(9.8 \%)$ \\
\hline Length of stay, mean (SD) & $5.74(4.60)$ & $8.92(6.45)$ & $11.12(9.73)$ \\
\hline Discharge destination: Back home & $381(78.6 \%)$ & $42(37.5 \%)$ & $5(9.8 \%)$ \\
\hline Stroke recurrence within 12 months & $66(13.6 \%)$ & $21(18.8 \%)$ & $11(21.6 \%)$ \\
\hline
\end{tabular}

Abbreviations: $N$ number, SD Standard deviation, NIHSS National Institute of Health Stroke Scale

supportive discharge beyond initial neurological deficits, like patients with the mentioned cardiovascular comorbidities. These findings demonstrate a possible clinical value of class-analyses. In case of more severely affected patients the best time point for discharge remains unclear and is supposedly influenced by multiple substantial medical reasons, such as complications of therapy [22]. 
Table 2 Factors predicting membership of the moderately compared to mildly affected class

\begin{tabular}{lll}
\hline $\mathbf{N}=\mathbf{5 9 5}$ & OR $(\mathbf{C l})$ & $\boldsymbol{P}$-value \\
\hline Age & $1.02(1.00,1.04)$ & 0.058 \\
Stroke subtype & $0.83(0.60,1.16)$ & 0.280 \\
Initial NIHSS & $1.74(1.29,2.35)$ & $<0.001$ \\
Length of initial inpatient stay & $1.08(1.04,1.12)$ & $<0.001$ \\
Prior myocardial infarction & $1.52(1.01,3.17)$ & 0.262 \\
Atrial fibrillation & $2.20(1.30,3.75)$ & 0.004 \\
Diabetes & $1.91(1.10,3.30)$ & 0.021 \\
\hline
\end{tabular}

The analysis of parameters distinguishing the classes of patients with moderately and severely decreased selfreported outcomes was limited by the smaller sample sizes with 122 and 51 patients assigned to these classes. Severity of neurological symptoms was identified as the only significant parameter differentiating between these two classes. However, living alone predicted assignment to the class with severely impaired health status in univariate analysis, and lack of significance after adjustment for cofactors might be related to sample size. The impact of living status on initial stroke severity, e.g., by delayed presentation of patients living alone, has been described previously [25], and the potential effect on further course of disease is discussed controversially [8, 12, 32]. Our data point towards a possible association of living alone with worse self-reported health status in moderately to severely affected stroke patients, but need to be verified in a larger cohort. The higher rate of endovascular treatment in the class of severe impairment is supposedly a cause of more severe stroke symptoms and large vessel occlusions in this group [29]. Though women were more frequent in the severely impaired class, sex did not significantly contribute to distinguish it from the class of moderately affected patients and we cannot confirm its negative influence on PROs described for mild to moderately affected outpatient stroke patients in our inpatient stroke center cohort [15].

One limitation of our study is the reduced generalizability due to the single-center design representing patients from a university stroke center located in an urban area. The large number of patients lost to

Table 3 Factors predicting membership of the severely compared to moderately affected class

\begin{tabular}{lll}
\hline $\mathbf{N = 1 5 8}$ & OR $(\mathbf{C l})$ & $\boldsymbol{P}$-value \\
\hline Age & $1.04(0.99,1.08)$ & 0.117 \\
Sex = Female & $1.75(0.68,4.52)$ & 0.247 \\
Initial NIHSS & $3.51(1.76,6.99)$ & $<0.001$ \\
Living alone & $1.52(0.61,3.79)$ & 0.371 \\
Endovascular thrombectomy & $1.85(0.70,4.94)$ & 0.216 \\
\hline
\end{tabular}

follow-up represents a further limitation. To assess the potential influence of missing follow-up data on the results, we performed a sensitivity analysis based on multiple imputation in a previous analysis. This analysis of the imputed full dataset revealed no substantial differences in outcome and predictive factors [27]. Another limitation concerns the comparison between the moderate and severe class, which might underestimate associations due to small class size of patients with severe impairment.

\section{Conclusions}

Altogether, our study demonstrates the possibility and potential clinical benefit of classifying patients in the course of acute stroke treatment by a standard set of PROMs for risk stratification. The differentiated view on patients separated by classes reveals differences in characteristics noticeable only in subgroups of outcome, relevant as potential driving factors of worse long-time outcome, and thus also as potential targets for new treatment approaches. The rarity of distinguishing characteristics shows the limits of a standard set. Class models from focused PROMs may represent the next step towards a more individually tailored treatment within the framework of value-based medicine.

\section{Abbreviations}

PROMs: Patient reported outcome measures; PROMIS: Patient-reported Outcomes Measurement Information System; PROMIS-10: PROMIS 10Question Short Form; ICHOM-SSS: International Consortium for Health Outcome Measurement Standard Set for Stroke; PHQ-4: Patient Health Questionnaire-4; PH: Physical health; MH: Mental health; Anx: Anxiety; DP: Depressive symptoms; AIS: Acute ischemic stroke; TIA: Transient ischemic attack; ICH: Intracerebral hemorrhage; NIHSS: National Institutes of Health Stroke Scale; ABIC: Adjusted Bayesian Information Criterion; VLMRLRT: Vuong-Lo-Mendell-Rubin likelihood ratio test; LMR-ALRT: LO-MendellRubin adjusted likelihood ratio test

\section{Supplementary Information}

The online version contains supplementary material available at https://doi org/10.1186/s42466-021-00146-9.

\section{Additional file 1.}

\section{Acknowledgements}

NA.

Disclosures

DLR, TS, LL, LK, CG, MH, and GT have no potential conflict of interest to report.

\section{Authors' contributions}

D. Leander Rimmele contributed to the acquisition, analysis and interpretation of the data and drafted the manuscript. Theresa Schrage contributed to the analysis of data and revised the manuscript for intellectual content. Lisa Lebherz revised the manuscript for intellectual content. Levente Kriston contributed to the conceptualization of the study, analysis and interpretation of the data, and revised the manuscript for intellectual content. Christian Gerloff contributed to the planning of the study, and the interpretation of the results and revised the manuscript for intellectual content. Martin Härter contributed to the conceptualization and 
the interpretation of results of the study, drafted the study design and revised the manuscript for intellectual content. Götz Thomalla contributed to the conceptualization and the interpretation of results of the study, drafted the study design and revised the manuscript for intellectual content. The author(s) read and approved the final manuscript.

\section{Funding}

This work is funded by the Innovation Fund of the German Federal Joint Committee with the grant number 01VSF16023. The German Federal Joint Committee reviewed and approved the study design during the grant application process. It had no role in the conduct of the study or publication process.

\section{Availability of data and materials}

Deidentified individual participant data will be made available upon reasonable request and where necessary after approval of the ethics committee by the corresponding author within 24 months after publication. Additional information can be taken from our study protocol.

\section{Declarations}

\section{Ethics approval and consent to participate}

The ethics committee of the Hamburg chamber of physicians approved the study protocol (PV54565).

\section{Consent for publication}

NA.

\section{Competing interests}

DLR, TS, LL, LK, and MH have nothing to report. CG reports personal fees from Amgen, Bayer Vital, Bristol-Myers Squibb, Boehringer Ingelheim, Sanofi Aventis, Abbott, and Prediction Biosciences outside the submitted work. GT reports receiving consulting fees from Acandis, grant support, and lecture fees from Bayer, lecture fees from Boehringer Ingelheim, Bristol-Myers Squibb/Pfizer, and Daiichi Sankyo, and consulting fees and lecture fees from Stryker outside the submitted work.

\section{Author details}

'Department of Neurology, University Medical Center Hamburg-Eppendorf, Martinistr. 52, 20246 Hamburg, Germany. ${ }^{2}$ Department of Medical Psychology, University Medical Center Hamburg-Eppendorf, Martinistr. 52, 20246 Hamburg, Germany.

Received: 28 June 2021 Accepted: 26 July 2021

Published online: 23 August 2021

\section{References}

1. Abdul-Rahim, A. H., Fulton, R. L., Sucharew, H., Kleindorfer, D., Khatri, P. Broderick, J. P.... Weimar, C. (2015). National institutes of health stroke scale item profiles as predictor of patient outcome: External validation on independent trial data. Stroke, 46(2), 395-400. https://doi.org/10.1161/ STROKEAHA.114.006837.

2. Baker-Goering, M. M., Howard, D. H., Will, J. C., Beeler Asay, G. R., \& Roy, K. (2019). Association between self-reported hypertension and antihypertensive medication use and cardiovascular disease-related events and expenditures among patients diagnosed with hypertension. Public Health Reports, 134(5), 493-501. https://doi.org/10.1177/0033354919864363.

3. Bråndal, A., Eriksson, M., Glader, E. L., \& Wester, P. (2019). Effect of early supported discharge after stroke on patient reported outcome based on the Swedish Riksstroke registry. BMC Neurology, 19(1), 1-11. https://doi.org/1 0.1186/s12883-019-1268-8.

4. Broomfield, N. M., Quinn, T. J., Abdul-Rahim, A. H., Walters, M. R., \& Evans, J. J. (2014). Depression and anxiety symptoms post-stroke/TIA: Prevalence and associations in cross-sectional data from a regional stroke registry. BMC Neurology, 14(1), 1-9. https://doi.org/10.1186/s12883-014-0198-8.

5. Cella, D., Ph, D., Riley, W., Stone, A., Rothrock, N., Yount, S., ... Rose, M. (2010). Initial adult health item banks and first wave testing of the patient reported outcomes measurement information system (PROMIS) network. Journal of Clinical Epidemiology, 63(11), 1179-1194. https://doi.org/10.1016/j. jclinepi.2010.04.011.Initial.
6. Dhamoon, M. S., Moon, Y. P., Paik, M. C., Sacco, R. L., \& Elkind, M. S. V. (2014). Diabetes predicts long-term disability in an elderly urban cohort: The northern Manhattan study. Annals of Epidemiology, 24(5), 362-368. https:// doi.org/10.1016/j.annepidem.2013.12.013.Diabetes.

7. Feigin, V., Barker-Collo, S., Parag, V., Senior, H., Lawes, C., Ratnasabapathy, Y., \& Glen, E. (2010). Auckland stroke outcomes study part 1: Gender, stroke types, ethnicity, and functional outcomes 5 years poststroke. Neurology, 75(18), 1597-1608. https://doi.org/10.1212/WNL. 0b013e3181fb44b3.

8. Gandhi, S., Goodman, S. G., Greenlaw, N., Ford, I., McSkimming, P., Ferrari, R., .. Udell, J. A. (2019). Living alone and cardiovascular disease outcomes. Heart, 105(14), 1087-1095. https://doi.org/10.1136/heartjnl-2018-313844.

9. Ganesh, A., Luengo-Fernandez, R., \& Rothwell, P. M. (2020). Late functional improvement and 5-year poststroke outcomes: A population-based cohort study. Journal of Neurology, Neurosurgery and Psychiatry, 91(8), 831-839. https://doi.org/10.1136/jnnp-2019-322365.

10. Heuschmann, P. U., Wiedmann, S., Wellwood, I., Rudd, A., Di Carlo, A., Bejot, Y., ... Wolfe, C. D. A. (2010). Three-month stroke outcome: The European registers of stroke (EROS) investigators. Neurology, 76(2), 159-165. https://doi. org/10.1212/wnl.0b013e318206ca1e.

11. International Consortium for Health Outcomes Measurement (ICHOM). (2017). Measuring results that matter Ability to communicate Stroke. Data Collection Reference Guide. http://www.Ichom.Org/Medical-Conditions/ Stroke, 32.

12. Ishikawa, Y., Hifumi, T., \& Urashima, M. (2020). Influence of living alone or with a spouse only on the short-term prognosis in patients after an acute ischemic stroke. International Journal of Environmental Research and Public Health, 17(21), 1-11. https://doi.org/10.3390/ijerph17218223.

13. Jaser, S. S., Whittemore, R., Choi, L., Nwosu, S., \& Russell, W. E. (2019). Randomized trial of a positive psychology intervention for adolescents with type 1 diabetes. Journal of Pediatric Psychology, 44(5), 620-629. https://doi. org/10.1093/jpepsy/jsz006.

14. Johnson, C. O., Nguyen, M., Roth, G. A., Nichols, E., Alam, T., Abate, D., Murray, C. J. L. (2019). Global, regional, and national burden of stroke, 19902016: A systematic analysis for the global burden of disease study 2016. The Lancet Neurology, 18(5), 439-458. https://doi.org/10.1016/S1474-4422(19)3 0034-1.

15. Katzan, I. L., Schuster, A., Bain, M., \& Lapin, B. (2019). Clinical symptom profiles after mild-moderate stroke. Journal of the American Heart Association, 8(11), 1-10. https://doi.org/10.1161/JAHA.119.012421.

16. Katzan, I. L., Schuster, A., Newey, C., Uchino, K., \& Lapin, B. (2018). Patientreported outcomes across cerebrovascular event types. Neurology, 91(23), e2182-e2191. https://doi.org/10.1212/wnl.0000000000006626.

17. Katzan, I. L., Thompson, N. R., Lapin, B., \& Uchino, K. (2017). Added value of patient-reported outcome measures in stroke clinical practice. Journal of the American Heart Association, 6(7), 1-10. https://doi.org/10.1161/JAHA.116.005356.

18. Katzan, I. L., Thompson, N. R., Uchino, K., \& Lapin, B. (2018). The most affected health domains after ischemic stroke. Neurology, 90(16), e1364e1371. https://doi.org/10.1212/wnl.0000000000005327.

19. Langhorne, P., Taylor, G., Murray, G., Dennis, M., Anderson, C., Bautz-Holter, E. , ... Wolfe, C. (2005). Early supported discharge services for stroke patients: A meta-analysis of individual patients' data. Lancet, 365(9458), 501-506. https://doi.org/10.1016/S0140-6736(05)70274-9.

20. Loupy, A., Loupy, A., Coutance, G., Bonnet, G., Bonnet, G., Van Keer, J., ... Kobashigawa, J. (2020). Identification and characterization of trajectories of cardiac allograft vasculopathy after heart transplantation: A populationbased study. Circulation, 1954-1967. https://doi.org/10.1161/CIRCULATIONA HA.119.044924.

21. Löwe, B., Wahl, I., Rose, M., Spitzer, C., Glaesmer, H., Wingenfeld, K., ... Brähler, E. (2010). A 4-item measure of depression and anxiety : Validation and standardization of the Patient Health Questionnaire-4 ( $\mathrm{PHQ}-4$ ) in the general population. Journal of Affective Disorders, 122(1-2), 86-95. https:// doi.org/10.1016/j.jad.2009.06.019.

22. Minaeian, A., Patel, A., Essa, B., Goddeau, R. P., Moonis, M., \& Henninger, N. (2017). Emergency department length of stay and outcome after ischemic stroke. Journal of Stroke and Cerebrovascular Diseases, 26(10), 2167-2173. https://doi.org/10.1016/j.jstrokecerebrovasdis.2017.04.040.

23. Oberski, D. (2016). Mixture models: Latent profile and latent class analysis. In J. Robertson, \& M. Kaptein (Eds.), Modern statistical methods for HCl. Humancomputer interaction series, (pp. 275-287). Springer. https://doi.org/10.1007/ 978-3-319-26633-6_12. 
24. Obura, M., Beulens, J. W. J., Slieker, R., Koopman, A. D. M., Hoekstra, T., Nijpels, G., ... Rutters, F. (2020). Clinical profiles of post-load glucose subgroups and their association with glycaemic traits over time: An IMIDIRECT study. Diabetic Medicine, (June), 1-10. https://doi.org/10.1111/ dme.14428.

25. Reeves, M. J., Prager, M., Fang, J., Stamplecoski, M., \& Kapral, M. K. (2014). Impact of living alone on the care and outcomes of patients with acute stroke. Stroke, 45(10), 3083-3085. https://doi.org/10.1161/STROKEAHA.114. 006520.

26. Reeves, M., Khoury, J., Alwell, K., Moomaw, C., Flaherty, M., Woo, D., ... Kleindorfer, D. (2013). Distribution of national institutes of health stroke scale in the Cincinnati/northern Kentucky stroke study. Stroke, 44(11), 32113213. https://doi.org/10.1161/STROKEAHA.113.002881.

27. Rimmele, D. L., Lebherz, L., Frese, M., Appelbohm, H., Bartz, H. J., Kriston, L., ... Thomalla, G. (2020). Health-related quality of life 90 days after stroke assessed by the international consortium for health outcome measurement standard set. European Journal of Neurology, 27(12), 2508-2516. https://doi. org/10.1111/ene.14479.

28. Rimmele, D. L., Lebherz, L., Frese, M., Appelbohm, H., Bartz, H.-J., Kriston, L., ... Thomalla, G. (2019). Outcome evaluation by patient reported outcome measures in stroke clinical practice (EPOS) protocol for a prospective observation and implementation study. Neurological Research and Practice, 0, 1-7. https://doi.org/10.1186/s42466-019-0034-0.

29. Saber, H., \& Saver, J. L. (2020). Distributional validity and prognostic power of the National Institutes of Health stroke scale in US administrative claims data. JAMA Neurology, 77(5), 606-612. https://doi.org/10.1001/jamaneurol.2 019.5061 .

30. Salinas, J., Sprinkhuizen, S. M., Ackerson, T., Bernhardt, J., Davie, C., George, M. G., ... Schwamm, L. H. (2016). An international standard set of patientcentered outcome measures after stroke. Stroke, 47(1), 180-186. https://doi. org/10.1161/STROKEAHA.115.010898.

31. Tomari, S., Magin, P., Lasserson, D., Quain, D., Valderas, J. M., Dewey, H. M., .. Levi, C. R. (2020). The characteristics of patients with possible transient ischemic attack and minor stroke in the hunter and Manning Valley regions, Australia (the INSIST study). Frontiers in Neurology, 11 (May), 1-7. https://doi. org/10.3389/fneur.2020.00383.

32. Wondergem, R., Pisters, M. F., Wouters, E. J., Olthof, N., De Bie, R. A., VisserMeily, J. M. A., \& Veenhof, C. (2017). The course of activities in daily living: Who is at risk for decline after first ever stroke? Cerebrovascular Diseases, 43(1-2), 1-8. https://doi.org/10.1159/000451034.

33. Yeoh, Y. S., Koh, G. C. H., Tan, C. S., Lee, K. E., Tu, T. M., Singh, R. Luo, N. (2018). Can acute clinical outcomes predict health-related quality of life after stroke: A one-year prospective study of stroke survivors. Health and Quality of Life Outcomes, 16(1), 1-9. https://doi. org/10.1186/s12955-018-1043-3.

\section{Publisher's Note}

Springer Nature remains neutral with regard to jurisdictional claims in published maps and institutional affiliations.

Ready to submit your research? Choose BMC and benefit from:

- fast, convenient online submission

- thorough peer review by experienced researchers in your field

- rapid publication on acceptance

- support for research data, including large and complex data types

- gold Open Access which fosters wider collaboration and increased citations

- maximum visibility for your research: over $100 \mathrm{M}$ website views per year

At BMC, research is always in progress.

Learn more biomedcentral.com/submissions 